\title{
Awareness of Nursing Staff and Patients Regarding Hospitals Accreditation
}

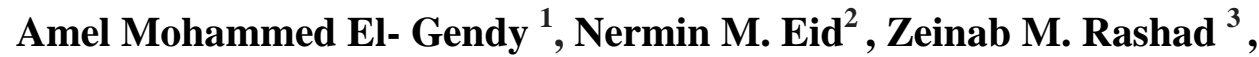 \\ Rehab Abd Allah Nassar ${ }^{4}$ \\ ${ }^{1}$ Nurse Specialist at Menoufia University Hospitals, ${ }^{2}$ Professor of Nursing Administration, \\ ${ }^{3}$ Lecturer of Nursing Administration, ${ }^{4}$ Lecturer of Nursing Administration, Faculty of Nursing- \\ Menoufia University
}

\begin{abstract}
Hospital accreditation is considered as an intervention performed to support patient safety and ensure high quality healthcare. Purpose: To assess the level of awareness of nursing staff and patients regarding hospital accreditation and determine the differences between the nursing staff awareness and patients' awareness regarding hospital accreditation. Design: Descriptive comparative design was utilized in this study. Setting: The study was conducted at ICU, surgical and medical departments at Menoufia University Hospitals, Shebin E Kom Teaching Hospital, National liver Institute and El Helal hospital. Sampling: A simple random sample of 448 staff nurses, 52 head nurses and 221 patients were included to participate in the study .Instruments: Nursing staff awareness and patients' awareness questionnaire. Results: the nursing staff had a moderate level of perception regarding hospital accreditation in El Helal Governmental hospital (81\%) and in National Liver Hospital (NLI)(60.4\%), On the other hand, nursing staff in Shebin Elkom Teaching Hospital (STH) and Menoufia University Hospitals(MUH) had a low level of perception . The nursing staff in all hospitals had not satisfied knowledge level and low level of awareness about hospital accreditation. Also, Patients in El Helal hospital had higher level of awareness and perception concerning than patients in Menoufia University hospital, Shebin ElKom Teaching hospital and National Liver Institute. El Helal patients had satisfied level of knowledge of hospital accreditation, while patients in National Liver Hospital (NLI), Shebin ElKom Teaching Hospital (STH) and Menoufia University Hospitals (MUH) had not satisfied level of knowledge in hospital accreditation. Conclusion: In the light of the present study results, the studied sample of the nursing staff had moderate level of perception of hospital accreditation in El Helal Governmental hospital and in National Liver Hospital (NLI).On the other hand, nursing staff in Shebin El-kom Teaching Hospital (STH) and Menoufia University Hospitals (MUH) had low level of perception and awareness of hospital accreditation. The patients in El Helal Governmental hospital had a moderate level of awareness, while patients in National Liver Hospital, Shebin ElKom Teaching Hospital and Menoufia University Hospitals had a low level of awareness regarding hospital accreditation. Recommendations: Healthcare organizations should develop awareness regarding hospital accreditation among nurses by integrating this concept into its core values and strategic management.
\end{abstract}

Key words: Awareness, hospital accreditation, nursing staff

\section{Introduction}

The nursing staff is the important human resources of the hospital that provide effective patient care and ensure that the patients receive satisfactory care quality (Yan and Kung, 2016). Nurse Managers are decision makers and they are seeking objective data for evaluating healthcare organizations. The nursing managers have direct link with multi professional actions .They make necessary strategies that help to perform changes that aim to implement hospital accreditation. They also have the responsibility of convincing the hospital managers to achieve and maintain hospital accreditation (Lucas et al., 2017).

Accreditation has been introduced as a systematic response to evaluate healthcare organizations. Accreditation standards are made to maintain continuous quality improvement efforts within accredited organization (Diab, 2011). Also, Accreditation is a great opportunity to enhance the power of 
some orders of nurses, help clarify rules and procedures by requiring them to be written down and new clinical protocols are implemented (Margaret Walton, 2020).

Also, Accreditation is a broad term that used in many sectors such as industry, education, air safety ,child care and health sector Accreditation is considered as a symbol of quality means that the hospitals achieve certain performance standards and evaluate their performance against national or international standards (Tabrizi et al., 2011). Hospital accreditation (HA) is currently used globally to ensure quality health services (Wang et al., 2015; Petereit, 2015). Numerous HA programs exist around the world; some are voluntary (e.g., in Jordan) and others are compulsory (e.g., in France). Most programs use the American Joint Commission accreditation standards (Sack, 2011).

Additionally, Hospital Accre-ditation uses self-assessment and external quality audits to check hospitals' compliance with standards (Brown, 2016) by identifying existing care practices that require reform or implementing new practices (Saif, 2016). Hospital accreditation ensures the systematic and strategic daily actions that result in the maintaince of the system in a given health organization (Lucas et al., 2017). Furthermore, Accreditation is considered as an intervention performed to support patient safety and ensure high quality healthcare (Brubakk et al., 2015). Hospital accreditation has become a symbol of honor for the hospitals (Yan and Kung, 2016). Hospital accreditation provides an effective and high reliable health care (Huang, 2018).

Healthcare providers agreed that the accreditation system is necessary and that accreditation improves patient safety. In addition, healthcare employees felt that accreditation increased interest and support for the improvement of the quality achieved by the leaders, the sense of community among the hospital staff, and the consideration and concern for one another (Park, 2017). Accreditation improves patient safety, including mortality, morbidity, and the incidence of infection (Shaw, 2014, Alkhenizan, 2011 and Thornlow, 2009).

Additionally, accreditation seems to have the potential to improve the quality of health services in fact, including hospital services. This is confirmed by studies conducted in several countries that point to improvements in the quality culture, strengthening of multidisciplinary team, positive standards of conformity of care related to adverse events, user-centered care and reduction of mortality in fully certified hospitals mortality in fully certified hospitals (Karlten, 2020 and EIJardali, 2017).

Meanwhile, Accreditation has many benefits such as increasing in team work. The staff is motivated to standardize clinical and administrative procedures, the staff learns from experiences of the reviewers, consequently, the accreditation leads to improvement in hospital image and staff satisfaction (Tabrizi, 2011). Hospital accreditation brings about positive standards of conformity of indicators related to adverse events, user - countered care and reduction of mortality in fully certified hospitals (Lucas et al., 2017).

The patients are considered as the clients of the health services and the target that the hospital aims to satisfy their needs. Hence; with the awareness of the patients of the accreditation, patients are be involved in their treatment decisions, they are be treated with dignity and respect, they express more satisfaction and they will make sure that their rights are protected (Devkaran , 2014). When the patients are aware of the hospital accreditation, they become aware of the care they receive and the rights and responsibilities they must have. The 
results, at the end, the patients become more satisfied.

\section{Significance of the study:}

Every hospital has standards of patient care which made by Ministry of Health and Population (MOHP) in Egypt in April 2013. It was supposed that the hospitals implemented these standards to evaluate the performance of the nursing staff and to meet patient needs.

From the researcher's clinical experience in hospitals, it was noticed that the hospital administration is not committed to implement the standards of care in different clinical areas. Consequently, nursing staff are not committed to follow nursing standards of care.

Accreditation is beneficial to the patients, health care providers and the hospital as accreditation improves patients care and outcomes. Accreditation encourages teamwork, collaboration, and motivation of the health care providers. Accreditation enables the hospital to better use its internal resources and enables the hospital to conduct changes.

So, it is important to conduct this study to assess the awareness of nursing staff and patients regarding hospital accreditation standards.

\section{Purpose of the study:}

To assess the level of awareness of nursing staff and patients regarding hospital accreditation and determine the differences between the nursing staff awareness and patients awareness regarding hospital accreditation.

\section{Research questions:}

- What is the nursing staff level of awareness regarding hospital accreditation at the previously mentioned hospitals?

- What is the patients level of awareness regarding hospital accreditation at the previously mentioned hospitals?

- What are the differences between the awareness of the nursing staff regarding hospital accreditation at the previously mentioned hospitals?

- What are the differences between the awareness of patients regarding hospital accreditation at the previously mentioned hospitals?

\section{Methods:}

Research design: A descriptive comparative research design was used to achieve the purpose of the study.

Settings: This study was carried out at ICU, medical and surgical departments in Menoufia University hospitals. Also, it was conducted in ICU, medical and surgical departments in Shebin Elkom Teaching Hospital. It was conducted in ICU, medical and surgical departments in the National Liver Institute (NLI). Besides, it was carried out in El-Helal Governmental Hospital. These hospitals lied in Shebin El-Kom.

Sampling: They consisted of two groups namely:

First group: A simple random sample of 448 staff nurses and 52 head nurses were included. A random sample was selected by the investigator from all departments and units from the previously mentioned hospitals.

Second group: simple random samples of 221 patients were included in this study. All patients were selected from general units. Because patients in critical care units had bad general conditions, so they were excluded, and the investigator had to replace them with conscious patients from general units. Each patient and nurse had the same code number.

Instruments: The data for this study was collected by using two different instruments developed by the investigator after reviewing the related literature:

Instrument one: Questionnaire about the awareness of the nursing staff regarding hospital. It was adapted from El-Sofany (2015). It was modified by the investigator. It included three parts: 
- Part one: Characteristics of respondents such as age, hospital, units, marital status, gender, years of experience, education, courses about hospital accreditation and attendance at accreditation program.

- Part two :Nurses perception questionnaire. It was used to measure nurses' perception (the nursing staff thoughts about accreditation) regarding hospital accreditation at the previously mentioned hospitals. It included three such as structure (11 items), process (14 items) and outcomes (11 items).

- Part three: Nurses knowledge Questionnaire. It was used to measure nurses' knowledge toward hospital accreditation standards at the previously mentioned hospitals. It included nine domains as follows: Patient rights and responsibilities standards (2 questions), patient access and assessment of patient standards (3 questions), providing care standards (4 questions), medication management standards (3 questions), patient safety, infection control and environmental safety standards(3 questions), information management standards(2 questions), performance improvement standards(2 questions), Organization management governance and leadership human services and nursing services standards(4 questions) and community involvement standards(2 questions).

Instrument two: Patient awareness Questionnaire regarding quality and hospital accreditation: It was developed by the investigator to assess the awareness regarding accreditation among patients at the study settings. It was designed based on the Egyptian health accreditation program (2013) and ElSofany (2015). It included three parts:

- Part one: Characteristics of respondents such as age, sex, and level of education.
- Part two: Patient's perception questionnaire regarding hospital accreditation at the previously mentioned hospitals. It included three components such as structure (9 items), process (21 items) and outcomes (6 items).

- Part three: Questionnaire to measure patient' knowledge toward hospital accreditation standards at the previously mentioned hospitals. It included nine domains as nurses knowledge questionnaire.

\section{Validity}

Data collection instruments were distributed to a Panel of Experts which consisted of five Assistant Professors in the field of Nursing Administration to judge the Content and Face validity of the instruments. All necessary modifications were done.

\section{Reliability of the instruments}

Coefficient alpha for nursing staff awareness scale was 0.81 and patient awareness scale was 0.84 .

\section{Pilot study}

Pilot study was conducted to assess the clarity and applicability of instruments and estimating the time needed for filling the form .The study was tested on $10 \%$ of total subjects, 45 staff nurses, 5 head nurses and 22 patients to evaluate the feasibility and clarity of instruments.

\section{Procedure}

An official letter is submitted from the Dean of the Faculty of Nursing to the directors of selected hospitals. It contained an explanation of the purpose and methods of data collection.

Before beginning to collect data from the study sample, the investigator introduced herself to them, explained the purpose of the study

Data was collected in the morning, afternoon and night shifts and studied sample filled the questionnaire in the presence of the investigator to ascertain that all questions were answered. The 
investigator asked nurses and head nurses to choose patient whom she cared to fill the questionnaire about patient. The time required for each nurse to fill the questionnaire was estimated to be 1520 minutes, whereas the time required for each patient to fill the questionnaire was estimated to be 25-30 minutes. Data was collected upon six months started from $1^{\text {st }}$ of June 2018 to $31^{\text {st }}$ of December 2018.

\section{Ethical considerations}

Approval of the Faculty of Nursing Ethical and Research Committee was obtained

The respondent rights were protected by ensuring voluntary participation, a written formal consent was obtained after explaining purpose, nature time of conducting the study, potential benefits of the study, how data will be collected, no invasive procedure, expected outcomes and the respondent rights to

\section{Results}

Table 1: Distribution of the Nursing Staff According to their Characteristics.

\begin{tabular}{|c|c|c|c|}
\hline \multicolumn{2}{|r|}{ Socio-demographic items } & No & $\%$ \\
\hline \multirow{4}{*}{ Hospital } & Menoufia University hospitals(MUH) & 199 & $39.8 \%$ \\
\hline & Shebin Elkom Teaching hospital(STH) & 138 & $27.6 \%$ \\
\hline & National Liver Institute(NLI) & 81 & $16.2 \%$ \\
\hline & El Helal(EH) & 82 & $16.4 \%$ \\
\hline \multirow{2}{*}{ Units } & Critical Care units & 279 & $55.8 \%$ \\
\hline & General Units & 221 & $44.2 \%$ \\
\hline \multirow{2}{*}{ Job } & Head nurse & 54 & $10.8 \%$ \\
\hline & Staff nurses & 446 & $892 \%$ \\
\hline \multirow{2}{*}{ Marital status } & Un married & 138 & $37.6 \%$ \\
\hline & Married & 362 & $72.4 \%$ \\
\hline \multirow{4}{*}{ Age } & From $20:$ less than 30years & 263 & $52.6 \%$ \\
\hline & From 30: less than 40 & 163 & $32.6 \%$ \\
\hline & From 40 : less than 50years & 37 & $7.4 \%$ \\
\hline & From 50 years and above & 37 & $7.4 \%$ \\
\hline \multirow{2}{*}{ Gender } & Male & 106 & $21.2 \%$ \\
\hline & Female & 394 & $78.8 \%$ \\
\hline \multirow{3}{*}{ Experiences } & From 1: less than 5 years & 96 & $19.2 \%$ \\
\hline & From 5: less than 10 years & 115 & $23 \%$ \\
\hline & From 10 : less than 15 years & 289 & $57.8 \%$ \\
\hline \multirow{4}{*}{ level of nursing education } & Nursing diploma & 66 & $13.2 \%$ \\
\hline & Associate degree in nursing & 302 & $60.4 \%$ \\
\hline & BSc in nursing & 120 & $24 \%$ \\
\hline & Others & 12 & $2.4 \%$ \\
\hline \multirow{2}{*}{$\begin{array}{l}\text { Attending courses about } \\
\text { accreditation }\end{array}$} & Yes & 75 & $15 \%$ \\
\hline & No & 425 & $85 \%$ \\
\hline
\end{tabular}

withdraw from the research study at any time in case of violation of their rights .

The respondent was assured that the data would be treated as strictly confidential; respondent and their anonymity would be maintained as they would not require mentioning their names.

\section{Statistical design:}

Data were statistically analyzed by using SPSS program version 22.

Descriptive Statistics: e.g. percentage $(\%)$, mean $(\bar{X})$ and standard deviation (SD) for demographic characteristic . Analytic statistics as Chi- square test $\left(\mathrm{x}^{2}\right)$ was used to study the association between two qualitative variables and Fisher exact test was used for smallest numbers. P- Value of $<0.05$ was considered statistically significant PValue of $<0.001$ was considered highly statistically significant. 
Table1 shows the characteristics nursing staff. As indicated in the table, about forty percent of the nursing staff worked in Menoufia University Hospitals (MUH), almost half of them worked in critical care units, more than three quarters were staff nurses, females and married. About half of them ranged from twenty to less than thirty years. More than half of them had ten to less than fifteen years of experience, graduated from associated degree of nursing and the majority of them $(85 \%)$ did not attend any accreditation course.

Table 2: Distribution of Patients According to their Characteristics.

\begin{tabular}{|c|c|c|c|}
\hline \multicolumn{2}{|r|}{ Demographic items } & No & $\%$ \\
\hline \multirow[t]{4}{*}{ Hospital } & $\begin{array}{l}\text { Menoufia University hospitals } \\
\text { (MUH) }\end{array}$ & 92 & $\underline{41.6} \%$ \\
\hline & Shebin Elkom Teaching hospital(STH) & 44 & $19.9 \%$ \\
\hline & National Liver Institute(NLI) & 21 & $9.5 \%$ \\
\hline & El Helal(EH) & 64 & $28.9 \%$ \\
\hline \multirow[t]{4}{*}{ Age } & less than 30 years & 56 & $25.3 \%$ \\
\hline & 30- less than 40 years & 53 & $23.9 \%$ \\
\hline & 40- less than 50 years & 61 & $27.6 \%$ \\
\hline & 50 years and above & 51 & $23.1 \%$ \\
\hline \multirow[t]{2}{*}{ Gender } & Male & 94 & $42.5 \%$ \\
\hline & Female & 127 & $\underline{\mathbf{5 7 . 5}} \%$ \\
\hline \multirow[t]{4}{*}{ Educational Level } & Primary school & 16 & $7.2 \%$ \\
\hline & Read and write & 18 & $8.1 \%$ \\
\hline & Secondary school & 9 & $4.1 \%$ \\
\hline & University degree & 178 & $\underline{80.5 \%}$ \\
\hline
\end{tabular}

Table 2 illustrates social characteristics of the studied patients. As indicated in the table, almost half of the studied patients were in Menoufia University Hospitals
(MUH). About one third of patients ranged from forty to less than fifty years. About half of them were females and the majority of them had university degree.

Table 3: level of Nursing Staff Perception of Hospital Accreditation.

\begin{tabular}{|c|c|c|c|c|c|c|}
\hline \multicolumn{2}{|c|}{ Settings } & \multicolumn{2}{|c|}{ Perception Level } & \multirow[t]{2}{*}{ Mean \pm SD. } & \multirow[t]{3}{*}{$\mathbf{F}$} & \multirow[t]{2}{*}{$\begin{array}{c}\text { p- } \\
\text { value }\end{array}$} \\
\hline \multirow{5}{*}{$\begin{array}{l}\text { Nursing staff } \\
\text { total perception }\end{array}$} & & Low \% & Moderate \% & & & \\
\hline & (MUH) & $37.4 \%$ & $0 \%$ & $26.9 \pm 15.9$ & & \multirow{4}{*}{$* 0$} \\
\hline & $(\mathrm{STH})$ & $57.2 \%$ & $0 \%$ & $41.2 \pm 16.9$ & \multirow{3}{*}{55} & \\
\hline & (NLI) & $0 \%$ & $61.9 \%$ & $44.6 \pm 14.9$ & & \\
\hline & $(\mathrm{EH})$ & $0 \%$ & $69 \%$ & $49.7 \pm 13.3$ & & \\
\hline
\end{tabular}

Highly statistically difference $(\mathbf{P}$ Value $\mathbf{= 0 . 0 0})$.

Table 3 showed level of nursing staff perception of hospital accreditation .It showed that El Helal nursing staff and National Liver Institute (NLI) nursing staff had moderate level of total perception regarding hospital accreditation, while nursing staff at Menoufia
University Hospitals (MUH) and Shebin El Kom Teaching Hospital (STH) nursing staff had low level of perception regarding hospital accreditation. There were highly statistically differences between nursing staff total perception among selected hospitals as ( $\mathrm{P}$ Value $=0.00)$. 
Table 4: Level of Patients Perception of Hospital Accreditation.

\begin{tabular}{|c|c|c|c|c|c|c||}
\hline \multirow{2}{*}{ Settings } & \multicolumn{3}{c|}{ Patient Perception Level } & \multirow{2}{*}{ Mean \pm SD } & \multirow{2}{*}{ F } \\
\cline { 3 - 6 } & Low \% & Moderate\% & High\% & & \\
\hline \multirow{3}{*}{$\begin{array}{c}\text { Patient total } \\
\text { perception }\end{array}$} & MUH & $\mathbf{5 3 . 8 \%}$ & $0 \%$ & $0 \%$ & $38.7 \pm 16.7$ & 24.1 \\
\cline { 2 - 7 } & STH & $0 \%$ & $60.4 \%$ & $0 \%$ & $43.5 \pm 13.9$ \\
\cline { 2 - 7 } & NLI & $55.5 \%$ & $0 \%$ & $0 \%$ & $39 \pm 11.5$ \\
\hline
\end{tabular}

Table 4 showed the level of patients' perception regarding hospital accreditation at selected study settings. It showed that El Helal patients had high level of perception regarding hospital accreditation, while Shebin El Kom Teaching Hospital (STH) patients had moderate level of perception. Menoufia University Hospitals (MUH) patients and National Liver Institute patients (NLI) had low level of perception regarding hospital accreditation among selected study settings.

Table 5: Level of Nursing Staff Knowledge Level about Hospital Accreditation.

\begin{tabular}{|c|c|c|c|c|c|c|}
\hline \multirow{2}{*}{\multicolumn{2}{|c|}{ Settings }} & \multicolumn{2}{|c|}{ Knowledge level } & Mean \pm SD & $\mathrm{F}$ & $\begin{array}{c}\text { p- } \\
\text { value }\end{array}$ \\
\hline & & Not satisfied \% & Satisfied \% & & & \\
\hline \multirow{4}{*}{$\begin{array}{c}\text { Knowledge } \\
\text { level }\end{array}$} & MUH & $28.9 \%$ & $0 \%$ & $14.4 \pm 3.1$ & \multirow{4}{*}{19.5} & \multirow{4}{*}{$* 0$} \\
\hline & STH & $33.2 \%$ & $0 \%$ & $16.6 \pm 3.9$ & & \\
\hline & NLI & $31.4 \%$ & $0 \%$ & $15.7 \pm 1.9$ & & \\
\hline & $\mathrm{EH}$ & $34 \%$ & $0 \%$ & $17 \pm 2.4$ & & \\
\hline
\end{tabular}

Highly statistically difference $(\mathbf{P}$ Value $\mathbf{= 0 . 0 0})$.

Table 5 showed the nursing staff level of knowledge about hospital accreditation at selected study settings. It showed that all selected hospitals were not satisfied knowledge level about hospital accreditation, while El Helal nursing staff had higher knowledge level about hospital accreditation as compared by Menoufia University Hospitals ( MUH) nursing staff. There were highly statistically significant difference between nursing staff knowledge level among selected hospitals as $(\mathrm{P}$ Value $=$ $0.00)$.

Table 6: The Level of Patient Knowledge Level about Hospital Accreditation.

\begin{tabular}{|c|c|c|c|c|c|}
\hline \multirow{2}{*}{\multicolumn{2}{|c|}{ Patient knowledge level }} & \multicolumn{2}{|c|}{ Patient Knowledge level } & \multirow{2}{*}{ Mean \pm SD } & \multirow{2}{*}{$\mathbf{F}$} \\
\hline & & Not satisfied\% & satisfied\% & & \\
\hline \multirow{4}{*}{ Knowledge level } & MUH & $38.7 \%$ & $0 \%$ & $9.7 \pm 4.1$ & \multirow{4}{*}{40} \\
\hline & $\mathrm{STH}$ & $41.6 \%$ & $0 \%$ & $10.4 \pm 4.4$ & \\
\hline & NLI & $36.9 \%$ & $0 \%$ & $9.2 \pm 4$ & \\
\hline & EH & $0 \%$ & $68 \%$ & $16.9 \pm 4.8$ & \\
\hline
\end{tabular}

Table 6 showed the patient knowledge level about hospital accreditation at selected study settings. It showed that El
Helal patients had satisfied knowledge level about hospital accreditation as compared by National Liver Institute (NLI) patients. 
Table 7: Level of Nursing Staff Total Awareness Level about Hospital Accreditation.

\begin{tabular}{|c|c|c|c|c|c|c|}
\hline \multirow{2}{*}{\multicolumn{2}{|c|}{$\begin{array}{l}\text { Nursing staff total } \\
\text { awareness }\end{array}$}} & \multicolumn{2}{|c|}{ Awareness level } & \multirow{2}{*}{ Mean \pm SD } & \multirow{2}{*}{$\mathbf{F}$} & \multirow{2}{*}{$\begin{array}{c}\text { p- } \\
\text { value }\end{array}$} \\
\hline & & Low\% & Moderate \% & & & \\
\hline \multirow{4}{*}{$\begin{array}{c}\text { Total } \\
\text { awareness }\end{array}$} & MUH & $33.90 \%$ & $0 \%$ & $41.4 \pm 17.5$ & \multirow{4}{*}{51.9} & \multirow{4}{*}{$* 0$} \\
\hline & STH & $47.37 \%$ & $0 \%$ & $57.8 \pm 20.4$ & & \\
\hline & NLI & $49.39 \%$ & $0 \%$ & $60.3 \pm 16.5$ & & \\
\hline & $\mathrm{EH}$ & $54.66 \%$ & $0 \%$ & $66.7 \pm 15.5$ & & \\
\hline
\end{tabular}

Highly statistically difference $(\mathbf{P}$ Value $\mathbf{= 0 . 0 0})$.

Table 7 showed the nursing staff total awareness about hospital accreditation at selected study settings. It showed that nursing staff at all selected hospitals had low level of total awareness regarding hospital accreditation, while El Helal nursing staff had higher level of total awareness regarding hospital

accreditation as compared by Menoufia University Hospitals (MUH) nursing staff. There were highly statistically significant difference between nursing staff total awareness level among selected hospitals as ( $\mathrm{P}$ Value $=0.00$ )

Table 8: Level of Patients' Awareness of Hospital Accreditation.

\begin{tabular}{|c|c|c|c|c|c||}
\hline \multirow{2}{*}{ Settings } & \multicolumn{2}{|c|}{ Patient awareness level } & \multirow{2}{*}{ Mean \pm SD } & \multirow{2}{*}{ F } \\
\cline { 2 - 5 } & Low\% & Moderate & & \\
\hline \multirow{3}{*}{$\begin{array}{c}\text { Patient total } \\
\text { awareness }\end{array}$} & MUH & $\mathbf{3 9 . 7 \%}$ & $0 \%$ & $48.4 \pm 20.5$ & \\
\cline { 2 - 5 } & STH & $44.2 \%$ & $0 \%$ & $53.9 \pm 17.9$ & \multirow{2}{*}{28.5} \\
\cline { 2 - 5 } & NLI & $40.3 \%$ & $0 \%$ & $49.2 \pm 15$ & \\
\cline { 2 - 5 } & EH & $0 \%$ & $\mathbf{6 1 . 6 \%}$ & $75.1 \pm 16.9$ & \\
\hline
\end{tabular}

Table 8 showed the patients awareness of hospital accreditation at selected study settings. It showed that patients in El Helal hospital had moderate

\section{Discussion}

Hospital accreditation has become a symbol of honor for the hospitals. It ensures the systematic and strategic daily actions that result in the maintenance of the system in each health organization. Accreditation is an effective tool for improving the quality of healthcare.

In relation to the nursing staff level of awareness, the results revealed that the nursing staff had low level of awareness about hospital accreditation in all hospitals. The studied nursing staff had moderate level of perception level of awareness regarding hospital accreditation as compared to Menoufia University Hospitals (MUH).

about hospital accreditation in $\mathrm{El}$ Helal Governmental hospital and in National Liver Hospital. Nursing staff in Shebin El Kom Teaching Hospital and Menoufia University Hospitals had low level of perception of hospital accreditation. The nursing staff in all hospitals had not satisfying knowledge level in hospital accreditation.

From the investigators' point of view, this result could be due to El Helal hospital is a small-sized hospital and follows the health insurance regimen 
which confirm the quality and Egyptian Accreditation Program, and National Liver Institute (NLI) is middle- sized hospital, while Menoufia University Hospitals (MUH) had lowest level of total awareness regarding hospital accreditation as MUH are considered the largest and main hospital in Menoufia Governate and the nursing staff had excessive workload.

The present study results were supported by El Jardali (2014) who made a study entitled the impact of accreditation of primary healthcare centers: successes, challenges and policy implications as perceived by healthcare providers and directors in Lebanon which confirmed that health care professionals in small and medium-sized hospitals have better awareness than in large-sized hospitals.

Also, these results are congruent with the study of Yeldiz (2014) about perceptions of nurses on the impact of accreditation on quality of care. They confirmed that the nurses who were working in university hospitals had the lowest awareness regarding accreditation. It is commonly appreciated that the personal rights (salary, post guarantee, etc.) of nurses working at university hospitals are of lower status than those nurses working at Ministry of Health Hospitals. Therefore, nurses working at university hospitals leave their institution if they find jobs at other hospitals.

Furthermore, results of this study are congruent with the study of Reisi (2018). It was entitled the impact of accreditation on nurses' perceptions of quality of care in Iran and its barriers and facilitators. It confirmed that financial and staff shortages, staff resistance, and heavy workload were previously reported as potential barriers to perceive accreditation in health care organizations. Other institutional barriers were lack of monitoring, weak communication, and lack of supportive organizational culture.

Also, this results was in agreement with the study of Diab (2011) about the perception of nurses and doctors on accreditation standards in Jordan. It was found that the participants had positive attitude regarding their perception of accreditation standards.

The results contradicted with the study of Alkhenizan and Shaw (2012) about the attitude of health care professionals towards accreditation: a systematic review of the literature. It showed that nurses' overall perceptions of care at the accredited hospitals increased significantly $(59 \%$ to $61 \%$ ), compared to the control hospitals (declined from $61 \%$ to $57 \%$ ).

Additionally, the results of this study contradicted with Shakibaie (2019) who showed that most large public hospitals (including university, military and social service hospitals) were more successful in process management than the small private hospitals. This is mostly attributable to the government funding received by the public hospitals. On the other hand, the total cost of pursuing accreditation is less than small hospitals

Also, these results were consistent with the study of Yeldiz (2014) namely Perceptions of nurses about the impact of accreditation on quality of care: A survey in a hospital in Turkey's revealed that these nurses were more frequently involved in efforts to practice accreditation (they were asked to take part in meetings and make decisions). On the other hand, analysis of the interviews 
indicated that those nurses without administrative duties viewed accreditation standards that required them to document what they do as "unnecessary paperwork" or waste of time.

These results are consistent with the study of El-Jardali (2017) about review of national policies and strategies to improve quality of health care and patient safety. Strong leadership and managerial support play a vital role in implementing an accreditation program and improving service quality.

These findings were in line with the results of Karimi et al (2015), about the Impact of hospitals accreditation on service delivery from the perspective views . They showed the positive effects of accreditation, such as improving service quality and infrastructure, activating hospital committees, creating performance guidelines, improving patients' education, and paying attention to staff members' continuous learning.

In a study by Pomey (2015) namely Accreditation and other external evaluation of quality and safety of care, who assessed the commonalities between the impacts and perceptions of accreditation in France and in Canada. According to the findings of this study, accreditation is a valuable opportunity to take stock of current practices and conduct inventory, it enhances the power of some orders of professionals, such as nurses, and helps clarify rules an and procedures by requiring them to be written down; new clinical protocols are implemented. On the other hand, resources for the implementation of changes are lacking, weak participation by doctors; selfassessment teams are not systematically designated as standing committees, lack of willingness on the part of upper management to pay greater attention to user satisfaction.

The present study results revealed that there was a highly positive statistically significant correlation between nursing staff total awareness and level of education and level of experience. This result was congruent with Aboelfotouh (2014), who study reported that there was highly statically significant relation between level of education and nursing staff total awareness and there wasn't any statically significant relation between nursing staff awareness and their job and gender.

Also, social characteristics of the studied patients included in the study sample $(n=221)$. Results showed that almost half of the studied patients were in Menoufia University Hospitals (MUH). About one third of patients ranged from forty to less than fifty years. About half of them were female and the majority of them had university degree.

Patients in El Helal hospital had higher level of awareness and perception concerning than patients in Menoufia University hospital, Shebin El-Kom Teaching hospital and National Liver Institute. El Helal patients had satisfied level of knowledge of hospital accreditation, while patients in National Liver Hospital (NLI), Shebin El-Kom Teaching Hospital (STH) and Menoufia University Hospitals (MUH) had not satisfied level of knowledge in hospital accreditation.

From the investigator point of view, this result may be due to El Helal patients worked in governmental institutions such as schools where there had accreditation. So, they had higher levels of perception and awareness. 
These results were consistent with the study of Yeldiz (2014) about perceptions of nurses on the impact of accreditation on quality of care, the examination of the survey for patient satisfaction showed that patient satisfaction increased after accreditation. This implies that the nurses' perceptions and the findings about the patients' views concord well with each other. Higher patient satisfaction after accreditation could also be considered a sign of external validity of the questionnaire used at the Hacettepe University Adult Hospital.

On the other hand, these results are contradictory with the study of Reisi (2018) about the impact of accreditation on nurses' perceptions of quality of care in Iran and its barriers and facilitators who confirmed that there is improving in patient care, standardizing the care process, patients and staff satisfaction, better financial performance, improving organizational communication (inter/intra) and information flows, professional development, creating a coherent organizational culture, the increase of patients' awareness, reform of hospital processes, and knowledge dissemination were mentioned as accreditation outcomes.

Also, these results are contradictory with the study of Moghadam (2018) namely hospital accreditation in Iran: a qualitative case study of Kerman hospitals who showed that there are increasing in patients' awareness, proper complaint handling, and the activation of hospital committees were among the strengths of the program.

The study results showed that there was highly statically significant relation between patient total awareness and age, education and gender.

This result was congruent with Sack (2011), who study reported that there was highly statically significant relation between patient's gender and patient total awareness.

\section{Conclusion}

In the light of the present study results, the studied sample of the nursing staff had moderate level of perception of hospital accreditation in El Helal Governmental hospital and in National Liver Hospital (NLI). On the other hand, nursing staff in Shebin Elkom Teaching Hospital (STH) and Menoufia University Hospitals (MUH) had low level of perception and awareness of hospital accreditation.

\section{Recommendation}

Based on the findings of the present study, the following recommendations are proposed as:

\section{The Practical level:}

- Healthcare organizations should develop staff awareness regarding hospital accreditation by integrating this concept into its core missions and develop it through strategic management.

- Healthcare organizations should plan to increase the patient awareness about hospital accreditation to be included in the hospital philosophy and mission.

- Nurse managers should provide appropriate and supporting structure to promote staff awareness.

- Encourage the nursing staff to engage in activities that include attending various conferences about hospital accreditation to exchange new information through 
professional journals and publications.

\section{At educational level:}

- Nursing schools are required to integrate hospital accreditation in their curriculum.

- Collaborate with continuing education department in healthcare organization to develop effective training program on concepts and guidelines of hospital accreditation and to provide training for nurses and nurse managers about policies and procedures of hospital accreditation and how to implement it.

- Plan for post- graduation diplomat about quality and hospital accreditation in nursing colleges.

- Healthcare organizations should provide opportunities for continuing education to lifelong learning as a part of accreditation.

\section{At research level:}

Further research studies should be conducted to identify other factors that impede and facilitate hospital accreditation in different healthcare sectors.

References:

Al Khani, R. (2015). Improving Waiting Time and Patients' Experience in a Medical Retina Clinic. Unpublished Master Thesis, Royal College of Surgeons in Ireland.

Alhaleel, Azari Sulaiman (2018) Implementing a national accreditation programme in Kuwaiti hospitals: understanding the impact, facilitators and barriers using a multiple methods approach. $\mathrm{PhD}$ thesis Institute of Health and Wellbeing College of
Medical, Veterinary \& Life Sciences University of Glasgow .

Alkhenizan, and Shaw, C. (2012). The attitude of health care professionals towards accreditation: a systematic review of the literature. Journal of family and community medicine; Vol. 19,Issue 2

Brown, J (2016), The Healthcare Quality Hand Book A professional Resource and study guide, 29th edition, Lippincott Company, NewYork.

Brubakk, K et al., (2015). A systematic review of hospital accreditation: the challenges of measuring complex intervention effects. BioMed Central Health services research. ; Vol. 15, No. 280

Devkaran, S (2014) International Healthcare Accreditation: An analysis of clinical quality and patient experience in the UAE. Edinburgh Business School. Heriot-Watt University. Ph.D.

Diab, S. (2011). The extent to which Jordanian doctors and nurses perceive the accreditation in private hospitals. International Journal of marketing studies.;Vol. 3,No.1

Egyptian HealthCare Accreditation Program Standards for Hospitals (2013) 2nd edition.

El-Jardali and F et al., (2017). A review of national policies and strategies to improve quality of health care and patient safety: a case study from Lebanon and 
Jordan.BMC Health

Services Research

Volume 17

El-Jardali, F et al., (2014). The impact of accreditation of primary healthcare centers: successes, challenges and policy implications as perceived by healthcare providers and directors in Lebanon. BMC Health Services Research, 14, 86.

El-Sofany , H .(2015). Nursing awareness regarding accreditation standards in Health Insurance Hospital in El-Mansoura. Faculty of Nursing. AinShams University. Master degree.

Huang, 2018. The Perceptions of Physicians and Nurses Regarding the Establishment of Patient Safety in a Regional Teaching Hospital in Taiwan

Karltun, Sanne, Aase, Andersonet al, 2020. Knowledge management infrastructure to support quality improvement: A qualitative study of maternity services in four European

countries.https://doi.org/1 0.1016/j.healthpol.2019.11. $\underline{005}$

Lucas, Harold, "Healthcare Facilities Management" (2017). Integrated Studies. 15.

https://digitalcommons. murraystate.edu/bis437/15

Park, J. H. (2017), Understanding of the new Korea Healthcare Accreditation System, J
Korean Med Assoc, 54 (2), 142.

Pelletier, M. (2011), Accreditation Guide for Hospitals, $4^{\text {th }}$ edition, Philadelphia, W.B. Saunders Company.

Petrusevska, A et al. (2015). Evaluating Perception of the healthcare providers concerning accreditation in healthcare organizations in the $\mathrm{R}$. Macedonia. Journal of US-China public administration; Vol. 12, No.6.

Pomey, M. P., LemieuxCharles, L., Champagne, F., Angus, D., Shabah, A. \& Contandriopoulos, A. P. 2010. "Does accreditation stimulate change? A study of the impact of the accreditation process on Canadian healthcare organizations". Implementation Science, 5,31

Sack, C., Lütkes, P., Günther, W., Erbel, R., Jöckel, K., Holtmann, G., (2011) Challenging the Holy Grail of Hospital Accreditation: A Inpatient Cross-sectional Study of Satisfaction in the Field of Cardiology, BMC Health Services Research, 10

Saif, N. (2016). Quality of Health Services and Patients' Satisfaction in Accredited and NonAccredited Hospitals. International Journal of Business and Management, 11(10), 298.

Shakibaei, E. (2019), "Role of a hospital accreditation program in developing a 
process management

system: A qualitative

study", International

Journal of Health Care

Quality Assurance, Vol. 32 No. 1, pp. 120-136.

https://doi. org/

10.1108/IJHCQA-01-2018-

$\underline{0002}$

Shaw, C. 2015. "Accreditation is not a stand-alone solution". Eastern Mediterranean Health Journal, 21, 226-232 .

Shaw, C. D., Braithwaite, J., Moldovan, M., Nicklin, W., Grgic, I., Fortune, T. \& Whittaker, S. 2013. "Profiling health-care accreditation

organizations: an international survey". International Journal for Quality in Health Care, 25, 222-231

Tabrizi, J. S., Gharibi, F. \& Wilson, A. J. 2011. "Advantages and disadvantages of health care accreditation models". Health promotion perspectives, 1 , 1.

Touati, N. \& Pomey, M.-P. 2009. "Accreditation at a crossroads: Are we on the right track?". Health Policy, 90, 156-165

Wong, J. D., Bajcar, J. M., Wong, G. G., Alibhai, S. M., Huh, J.-H., Cesta, A., Pond, G. R. \& Fernandes, O. A. 2015. "Medication reconciliation at hospital discharge: evaluating discrepancies". Annals of Pharmacotherapy, 42, 1373-1379

Yan, Y and Kung, C. (2016). Investigation of hospital accreditation awareness and organizational learning promotion from nursing staff perspective. Arabian Journal of business and management review.; Issn:2223-5833.

Yildiz, A\& Kaya, S. (2014). Perception of nurses on the impact of accreditation on quality of care. Clinical Governance: An International Journal; Vol. 19, No.2.

Yildiz, A. \& Kaya, S. 2014. "Perceptions of nurses on the impact of accreditation on quality of care: A survey in a hospital in Turkey". Clinical Governance: an International Journal, 19, 69-82. 